\title{
Identification and expression analysis of the sucrose synthase gene family in pomegranate (Punica granatum L.)
}

\author{
Longbo Liu ${ }^{1}$, Jie Zheng ${ }^{\text {Corresp. } 1}$ \\ ${ }^{1}$ School of Life Science, Huaibei Normal University, Huaibei, Anhui, China \\ Corresponding Author: Jie Zheng \\ Email address: Zhengj@chnu.edu.cn
}

Background. Sucrose synthase (SUS, EC 2.4.1.13) is one of the major enzymes of sucrose metabolism in higher plants. It has been associated with $\mathrm{C}$ allocation, biomass accumulation, and sink strength. The SUS gene families have been broadly explored and characterized in a number of plants. The pomegranate (Punica granatum) genome is known, however, it lacks a comprehensive study on its SUS genes family.

Methods. PgSUS genes were identified from the pomegranate genome using a genome-wide search method. The PgSUS gene family was comprehensively analyzed by physicochemical properties, evolutionary relationship, gene structure, conserved motifs and domains, protein structure, syntenic relationships, and cis-acting elements using bioinformatics methods. The expression pattern of the PgSUS gene in different organs and fruit development stages were assayed with RNA-seq obtained from the NCBI SRA database as well as real-time quantitative polymerase chain reaction (qPCR).

Results. Five pomegranate SUS genes, located on four different chromosomes, were divided into three subgroupsaccording to the classification of other seven species. The PgSUS family was found to be highly conserved during evolution after studying the gene structure, motifs, and domain analysis. Furthermore, the predicted PgSUS proteins showed similar secondary and tertiary structures. Syntenic analysis demonstrated that four PgSUS genes showed syntenic relationships with four species, with the exception of PgSUS2. Predictive promoter analysis indicated that PgSUS genes may be responsive to light, hormone signaling, and stress stimulation. RNA-seq analysis revealed that PgSUS1/3/4 were highly expressed in sink organs, including the root, flower, and fruit, and particularly in the outer seed coats. qPCR analysis showed also that PgSUS1, PgSUS3, and PgSUS4 were remarkably expressed during fruit seed coat development. Our results provide a systematic overview of the PgSUS gene family in pomegranate, developing the framework for further research and use of functional PgSUS genes. 
1 Identification and expression analysis of the sucrose

2 synthase gene family in pomegranate (Punica

3 granatum L.)

4 Longbo Liu ${ }^{1}$, and Jie Zheng ${ }^{1, *}$

$5{ }^{1}$ School of Life Science, Huaibei Normal University, Huaibei, Anhui, China

6

7 Corresponding author:

8 Jie Zheng ${ }^{1}$

9 Dongshan road num 100, Huaibei, Anhui, 235000, China

10 Email address: Zhengi@,chnu.edu.cn 


\section{Abstract}

Background. Sucrose synthase (SUS, EC 2.4.1.13) is one of the major enzymes of sucrose metabolism in higher plants. It has been associated with $\mathrm{C}$ allocation, biomass accumulation, and sink strength. The $S U S$ gene families have been broadly explored and characterized in a number of plants. The pomegranate (Punica granatum) genome is known, however, it lacks a comprehensive study on its $S U S$ genes family.

Methods. $P g S U S$ genes were identified from the pomegranate genome using a genome-wide search method. The PgSUS gene family was comprehensively analyzed by physicochemical properties, evolutionary relationship, gene structure, conserved motifs and domains, protein structure, syntenic relationships, and cis-acting elements using bioinformatics methods. The expression pattern of the PgSUS gene in different organs and fruit development stages were assayed with RNA-seq obtained from the NCBI SRA database as well as real-time quantitative polymerase chain reaction (qPCR).

Results. Five pomegranate $S U S$ genes, located on four different chromosomes, were divided into three subgroupsaccording to the classification of other seven species. The PgSUS family was found to be highly conserved during evolution after studying the gene structure, motifs, and domain analysis. Furthermore, the predicted PgSUS proteins showed similar secondary and tertiary structures. Syntenic analysis demonstrated that four PgSUS genes showed syntenic relationships with four species, with the exception of PgSUS2. Predictive promoter analysis indicated that $P g S U S$ genes may be responsive to light, hormone signaling, and stress stimulation. RNA-seq analysis revealed that $P g S U S 1 / 3 / 4$ were highly expressed in sink organs, including the root, flower, and fruit, and particularly in the outer seed coats. qPCR analysis showed also that PgSUS1, PgSUS3, and PgSUS4 were remarkably expressed during fruit seed coat development. Our results provide a systematic overview of the PgSUS gene family in pomegranate, developing the framework for further research and use of functional PgSUS genes.

\section{Introduction}

Sucrose is the most common form of carbohydrate produced by photosynthetic leaves. It is imported into non-photosynthetic organs (sink organs) through the phloem (Lutfiyya et al., 2007). Sucrose has been acknowledged as a valuable carbon and energy source for various metabolic pathways related to plant growth and development, such as cell division, vascular tissue differentiation, seed germination, flowering induction, fruit development, anthocyanin synthesis, storage products accumulation, biotic and abiotic stresses response, and damage recovery (Wang et al., 2015). Therefore, the study of sucrose metabolism is beneficial for understanding numerous aspects of plant physiology. 
Sucrose synthase (SUS) and invertase (INV) are widely regarded as two key enzymes for the sucrose cleavage reaction. INV catalyzes the irreversible hydrolyzation of sucrose into glucose and fructose (Hirose et al., 2008), whereas SUS catalyzes the reversible cleavage of sucrose using uridine diphosphate (UDP) to yield fructose and UDP-glucose (Stein \& Granot, 2019). These enzymes are tightly linked with phloem sucrose unloading (Wang et al., 2015). SUS activity is highly associated with $\mathrm{C}$ allocation, biomass accumulation, and sink strength (Stein \& Granot, 2019). For instance, the deletion or suppression of the SUS gene decreases maize seed weight (Chourey et al., 1998), reduces pea seed mass (Craig et al., 1999), leads to tomato fruit setting abnormality (D'Aoust et al., 1999), inhibits stem thickening in Populus tomentosa (Li et al., 2020), and reduces the stem height, diameter, and biomass in aspen (Dominguez et al., 2021). The overexpression of $S U S$ increases the growth rate and facilitates plant biomass accumulation in Arabidopsis ( $X u \&$ Joshi, 2010), promotes cellulose biosynthesis and increases the lodging resistance in tobacco stem (Wei et al., 2015), and accelerates vegetative growth, thickens the secondary cell wall, and increases the stem breaking force in poplar (Li et al., 2019). SUS also plays important roles in sugar metabolism during fruit development. Citrus CitSus1 and CitSus2 (Islam et al., 2019), peach PpSUS1, PpSUS3, and PpSUS5 (Zhang et al., 2015), pear PbrSUS2 and PbrSUS15 (Lv et al., 2018), and apple MdSUS1s and MdSUS2.1 (Tong et al., 2018) are all thought to be responsible for the sucrose download and partitioning in fruits. Strawberry fruits with the suppression of FaSUS1 showed significantly delayed fruit ripening, and downregulated sucrose and anthocyanin contents (Zhao et al., 2017). Additionally, the SUS enzyme is thought to participate in the regulation of several important metabolic processes, such as cellulose and callose synthase, nitrogen fixation, abiotic stresses response, and development of shoot apical meristem (Stein \& Granot, 2019).

Sucrose synthase is encoded by a small, multigene family in both monocot and dicot species. The number of $S U S$ gene family members to date differs among the plant species. In maize, only three SUS genes have been identified (Duncan et al., 2006), however, five SUS genes have been found in grape (Zhu et al., 2017). Arabidopsis, rice, cacao, peach, tomato, and citrus all contain a SUS genes family with six SUS genes (Baud et al., 2007; Hirose et al., 2008; Li et al., 2015; Zhang et al., 2015; Goren et al., 2017; Duan et al., 2021; Islam et al., 2019), whereas seven, 11, 14, and 15 SUS genes were found in cotton (Chen et al., 2012), apple (Tong et al., 2018), Indian mustard (Koramutla et al., 2019), and poplar (An et al., 2014), respectively. In all cases, SUS genes showed structural conservation but functional divergence during evolution according to the physical and chemical properties of gene and protein structures, phylogenetic relatedness, and spatial-temporal expression patterns (Xu et al., 2014). The SUS gene family has been extensively studied in various plants. However, the $S U S$ genes in pomegranate not yet been described. 
Pomegranate (Punica granatum L.) is an ancient perennial plant species of the Punicaceae family that has become an emerging edible fruit crop due to its good environmental adaptation and wide medicinal applications (Conidi et al., 2020). The global pomegranate market is promising, with an expected $14 \%$ annual growth rate, and is expected to reach 23.14 billion United States dollars (USD) by year 2026 (Conidi et al., 2020). Improving the fruit quality is important to enhance the market competitiveness of pomegranate production. Particularly, the accumulation of sugar content is key in determining the taste, flavor, and value for most fleshy fruit crops ( Li et al., 2012). Therefore, the comprehensive analysis of sucrose synthase genes may improve the understanding of its molecular function and identify the key genes involved in pomegranate fruit sugar metabolism. Recently, the high-quality genome data of several cultivars of pomegranate have been released, including those of 'Dabenzi', 'Taishanhong', and 'Tunisia', which supplies genome data for further the molecular function identification of pomegranate genes (Qin et al., 2017; Yuan et al., 2018; Luo et al., 2020). Here, we identified and characterized five SUS genes on the pomegranate genome-wide scale and investigated their expression patterns. This study focused on PgSUS member isolation and identification, evolutionary relationships, exon/intron arrangement, conserved motif and domain, protein structure, synteny relationship, promoter elements, and expression patterns of the pomegranate $S U S$ gene family. These results will provide insight for further investigations of the possible functions of the $S U S$ gene family in pomegranate for regulating plant growth, particularly in the development and maturation of the fruit.

\section{Materials \& Methods}

\section{Obtaining genome sequences and identifying PgSUS family members in pomegranate}

The genome sequences and annotation data of pomegranate cv. Tunisia were obtained from the NCBI genome database

(https://www.ncbi.nlm.nih.gov/genome/13946?genome_assembly_id=720008) (Luo et al., 2020). Six known AtSUS proteins sequences were downloaded from TAIR database (http://www.arabidopsis.org/) and were used as a query to search against the pomegranate protein database (e-value $<1 \times 10^{-5}$, identify $>50 \%$ ). The search used a local BLAST alignment in order to identify potential members of SUS gene family in pomegranate. The hidden Markov model (HMM) profiles of the sucrose synthase domain (PF00862) and glycosyl transferases domain (PF00534) collected from the Pfam website (http://pfam.xfam.org/) were used as queries to search the candidate PgSUS from pomegranate proteins using HMMER 3.1 (e-value $<1 \times 10^{\text {- }}$ ${ }^{5}$ ) (Finn et al., 2015). The sucrose phosphate synthase (SPS) gene family with a sucrosephosphatase domain (PF05116) in the N-terminal was also found to contain SUS protein conserved domains (PF00862 and PF00534). The resulting putative proteins were further 
117 examined by using the SMART and NCBI conserved domain database (CDD) (Letunic \& Bork,

118 2018; Lu et al., 2020). We filtered out the candidates with a sucrose-phosphatase domain and

119 those that lacked the sucrose synthase and glycosyl transferases domains.

120 The information on the pomegranate SUS chromosomal positions was obtained from the

121 genome annotation data. The ExPasy website (http://web.expasy.org/protparam/) was used to

122 evaluate the molecular weight (MW), isoelectric point (pI), instability index, aliphatic index, and

123 grand average of hydropathicity (GRAVY). The NetPhos 3.1 server

124 (http://www.cbs.dtu.dk/services/NetPhos/) was used to predicted the PgSUS proteins

125 phosphorylation sites (Blom et al., 2004).

126 Nucleotide and amino acid sequences alignment of $\boldsymbol{S} \boldsymbol{U} \boldsymbol{S}$ genes from eight species

127 The nucleotide and proteins sequences of 68 SUS genes were collected from Arabidopsis

128 thaliana (6), Oryza sativa (6), Glycine max (12), Malus domestica (11), Pyrus bretschneideri

129 (17), Prunus persica (6), Vitis vinifera (5), and Punica granatum (5), respectively. Multiple SUS

130 genes sequence alignments were performed using the CLUSTAL_X program

131 (http://www.clustal.org/).

132 Phylogenetic analysis and classification of SUS gene family

133 A phylogenetic tree of 68 SUS proteins from eight species was generated using MEGA X

134 software (http://www.megasoftware.net/). The tree was based on the maximum-likelihood (ML)

135 method with the substitution model JTT $+\mathrm{G}+\mathrm{I}$ and 1,000 bootstrap replications. PgSUS proteins

136 were further categorized into different subfamilies according to the classification records of

137 subfamily members of other species. The proteins sequences used in the phylogenetic analysis

138 are listed in Data S1.

139 Gene structure construction, conserved motif, domain, and protein structure analysis

140 The information on gene structure for each of the 68 SUS genes was extracted from their GFF3

141 files. This data included sequence length, number, and arrangement of exons and introns. The

142 conserved motif type and sequence of the SUS family were analyzed by MEME (http://meme-

143 suite.org/tools/meme). The phmmer protein database

144 (https://www.ebi.ac.uk/Tools/hmmer/search/phmmer) was used to annotate the MEME motifs.

145 The conserved domains of the SUS proteins were determined using SMART (http://smart.embl-

146 heidelberg.de/). The gene structure, MEME, and conserved domain results were plotted with

147 TBtools (Chen et al., 2020). Secondary and tertiary structures of PgSUS proteins were predicted

148 using NPS@: SOPMA (https://www.predictprotein.org/signin) and the ExPaSy Swiss-Model

149 online software (http://swissmodel.expasy.org), respectively.

150 Syntenic analysis with four other species 
151 MCScanX was used to obtain the syntenic relationships of five species: Arabidopsis thaliana, 152 Malus domestica, Pyrus bretschneideri, Vitis vinifera, and Punica granatum (Wang et al., 2012).

153 The results were presented with TBtools (Chen et al., 2020).

\section{Cis-acting element analysis of $\mathrm{PgSUS}$ genes promoter regions}

We extracted 2,000 bp gene sequences of genomic DNA sequences upstream of the initiation codon (ATG). These were used to predict the putative cis-acting elements using PlantCARE online software (http://bioinformatics.psb.ugent.be/webtools/plantcare/html/).

\section{Expression pattern analysis of candidate PgSUS genes in pomegranate}

Two published sets of transcriptome data were used to investigate the expression characteristics of the PgSUS genes. The abundance of the PgSUS transcripts of 12 samples, including root, leaf, flower, and three different development stages of the pericarp, inner, and outer seed coats (50, 95, and 140 days after flowering, DAF), were collected from the NCBI Sequence Read Archive database (accession number SRP100581) (Qin et al., 2017). The expression profiles of the PgSUS genes were analyzed at different developmental stages of the seed coats in pomegranate cultivars 'Dabenzi' and 'Tunisia'. These were collected at 50, 95, and 140 DAF and three biological replicates were collected per sample for RNA sequencing (accession number SRP212814, Qin et al., 2020). Clean reads of each sample were aligned to the pomegranate reference genome by HISAT2, using default parameter settings (Kim et al., 2019) after conducting a quality assessment of the filtered reads using Trimmomatic (Bolger et al., 2014). The mapped reads assembly of each sample was completed using StringTie (Pertea et al., 2015). The different gene expression levels were calculated according to transcripts per kilobase of exon model per million mapped reads (TPM). The TPM value was transformed into $\log _{2}(\mathrm{TPM}+$ 1). The heatmap of the PgSUS genes expression was plotted using TBtools (Chen et al., 2020).

\section{Plant material}

Samples were collected from three-year-old 'Tunisia' pomegranate trees at $26^{\circ} \mathrm{C}$ under long-day conditions (14-h light/10-h dark) at approximately 60-70\% humidity conditions. The trees were cultivated at the horticultural experimental station of Huaibei Normal University. We collected young root, mature leaves, and flowers. Healthy, uniform fruits were randomly collected at 45, 75,115 , and $150 \mathrm{DAF}$, respectively. Three replicates were prepared for each stage and each replicate contained 15 fruits. The fruit pericarp and seed coat were separated by hand. All samples were collected and immediately frozen in liquid nitrogen and stored at $-80{ }^{\circ} \mathrm{C}$.

\section{Total RNA isolation and quantitative PCR expression assay}

Approximately $1 \mu \mathrm{g}$ of high quality RNA per sample was extracted using plant RNA extraction kits (TIANGEN, China). The first strand of cDNA synthesis was performed using the TIANScript II RT kit (TIANGEN, China). We diluted $20 \mu \mathrm{L}$ of cDNA from each sample to a total volume of $200 \mu \mathrm{L}$ using DEPC water. These were used as qPCR templates. The reaction 
187 mixture contained $1 \mu \mathrm{L}$ cDNA, $0.5 \mu \mathrm{L}$ each of the forward- and reverse-specific primer, $10 \mu \mathrm{L}$ 188 chamQ SYBR qPCR Master Mix (Vazyme, China), and $8 \mu \mathrm{L}$ DEPC water, for a total volume 20 $189 \mu \mathrm{L}$. The qPCR reaction was conducted in an ABI 7300 Real-Time PCR system with the 190 following amplification program: $95^{\circ} \mathrm{C}$ for $5 \mathrm{~min}, 40$ cycles of $95^{\circ} \mathrm{C}$ for $5 \mathrm{~s}$, and $60{ }^{\circ} \mathrm{C}$ for $35 \mathrm{~s}$.

191 The pomegranate PgActin gene served as the reference gene, and the relative expressions levels 192 of the genes were calculated according to Livak \& Schmitten (2001). Each sample was quantified in triplicate. Data were analyzed using SPSS software (22.0, USA) and Excel. All primers used for qPCR assay are shown in Data S2.

\section{6}

197

\section{Results}

\section{Identification of $\mathrm{PgSU}$ S genes}

Two searches were performed to identify all possible SUS family members in the pomegranate genome. We obtained 14 putative PgSUS candidates by local Blast alignment according to query sequences of six Arabidopsis SUS proteins. Then, 19 PgSUS candidates were scanned from the pomegranate genome database based on the HMMER search. These two methods identified a total of $14 \mathrm{PgSUS}$ candidates without a sucrose-phosphatase domain, which were verified with SMART and NCBI CDD databases. We found that $14 \mathrm{PgSUS}$ candidates belonged to five genes and determined that each gene two-to-four transcripts after extraction and comparing the generic feature formats of these candidates. Finally, the five longest transcripts were isolated as the representative genes and were named PgSUS1 to PgSUS5, according to their chromosomal information (Table 1).

Five PgSUS were dispersed on four chromosomes (Table 1). Among them, one single SUS gene originated from $\mathrm{Chr} 2,4$, and 6 respectively, while the rest two were located in Chr8. cDNA length analysis of five PgSUS genes revealed variations from 4,249 bp (PgSUS2) to 7,426 bp (PgSUS3). However, the coding DNA sequence (CDS) lengths were similar, and ranged from approximately 2,418 bp (PgSUS4) to 2,706 bp (PgSUS5). Their proteins were composed of 805901 amino acids, the putative molecular weights (MW) ranged from $92.26 \mathrm{kDa}$ to $102.58 \mathrm{kDa}$, and the theoretical isoelectric points $(\mathrm{pI})$ were approximately 5.99 to 8.19 . The instability index of the five PgSUS proteins ranged between 32.35 and 42.23. The aliphatic index (A.i) were between 81.60 and 92.87 and all of the PgSUS proteins were hydrophilic (Table 1). Our prediction of the phosphorylation sites in PgSUSs showed that serine was the most common site for phosphorylation Tow typically serine phosphorylation sites were observed in all PgSUS proteins (Data S3).

The ClustalW2 program was used to align the nucleotide/amino acid sequences of five pomegranate SUS and 63 SUS members with seven other species. The comparison results showed that these 68 genes shared a high sequence homology at the nucleotide level (average 
$22265.93 \%$ identity) as well as the protein level (average 65.16\% identity) (Data S4). Among the 223 five $P g S U S$ genes, the nucleotide and amino acid sequences of PgSUS1 were more similar to PgSUS4and their identity scores reached $80.98 \%$ and $89.94 \%$, respectively. PgSUS1 also showed similarity with $P g S U S 3$ with the sequence comparison scores of nucleotide and amino acid sequences of $68.03 \%$ and $69.44 \%$, respectively. A pair of PgSUS genes (PgSUS2-PgSUS5) were also observed to be closely related (68.51\% and $70.96 \%$ respectively) (Data S4). Phylogenetic analysis of $S U S$ family members

We used five PgSUS from pomegranate, six AtSUS from Arabidopsis, and 57 other SUS proteins to construct the phylogenetic tree in order to clarify the evolutionary relationships. A total of 68 SUS proteins results from phylogenetic analysis were classified into three distinct subgroups categorized as SUS I, SUS II, and SUS III (Fig. 1). Corresponding to the nucleotide/amino acid sequence identity (Data S4), PgSUS1 was clustered with PgSUS4 to form the SUS I branch, which contained well-characterized SUS genes including AtSUS1/2, PpSUS1/2/15, and VvSS4. PgSUS3 belonged to SUS II, which included MdSUS2.1 and VvSS3. Compared with the SUS I and SUS II subgroups, the genes clustered in the SUS III subgroup typically contained the proteins with a C-terminal extension, such as PgSUS2/5, AtSUS5/6 and MdSUS3.1/3.2/3.3 (Data S5). The results showed that although these $S U S$ family genes shared high sequence similarities, including five pomegranate $S U S$ genes, diversification was identified in this family through phylogenetic analysis.

\section{Gene structure, conserved motif, and domain analysis of $S U S$ family genes}

We further investigated the exons/introns exon/intron structure of all SUS genes to better understand the molecular evolution mechanism. These included five in pomegranate and 63 in other seven species according to the gene annotation files (Fig. 2A). SUS family gene sequences were split into approximately 15 exons in SUS I, and 14 exons in SUS II and SUS III genes, respectively, after taking introns loss into account (Fig. 2A; Data S6) (Xu et al., 2014). The nucleotide sequences of 68 SUS genes showed high similarity (Data S4), therefore, high conservation of these gene structures was expected. Exons with lengths of 152/155, 193, 177/174/129, 117, 167 and 225, were highly conserved and arranged in same order in the CDS regions of all three $S U S$ subgroups (Data S6). For each $S U S$ subgroup, the gene structure also showed unique features: compared with SUS II genes, intron loss was a common phenomenon in SUS I and SUS III genes (Data S6). In the SUS I subgroup, exons with lengths of 336, 432, and 564, were split into two (119 and 217), three (119, 217 and 96) and two (322 and 245) exons in the SUS II subgroup, respectively. In the SUS III subgroup, exons with lengths of 567, were split into two exons (322 and 245) in the SUS II subgroup (Data S6). The exon sizes and splitting varied among the $3^{\prime}$ end of the genes of the SUS III subgroup. This was associated with the 3' extension of SUS III proteins (Data S5; Data S6). In the SUS genes of pomegranate, the exons 
with lengths of 336 (or spilt into 119 and 217), 96, and 139 were typically conserved in PgSUSs (Data S4; Data S7). Moreover, in the same group, PgSUS genes showed a similar exon number, arrangement, and length with SUS genes from Arabidopsis (Baud et al., 2004), apple (Tong et al., 2018), grape (Zhu et al., 2017), peach (Zhang et al., 2015), pear (Lv et al., 2018), soybean (Xu et al., 2014), and rice (Hirose et al., 2008) (Fig. 2A; Data S6; Data S7).

We used the MEME online server to predict 15 motifs in the SUS gene family (Fig. 2B). Detailed information of these motifs is shown in Data S8. Among these motifs, the motifs 1, 3, 5, $6,9,10,11,12$, and 13 represented the sucrose synthase domain; motifs 2 and 7 corresponded to the glycosyl-transferase domain, and the motif feature of motifs 4,8 , and 15 were unknown (Data S8). The majority of the SUS proteins from eight species contained the 14 predicted motifs, except motif 14, and showed a consistent array (Fig. 2B). Motifs 2 and 7, as the elements of the glycosyl-transferase domain signature, were highly conserved, suggested that these motifs are essential for enzyme function of sucrose synthase. However, several motifs which corresponded to the sucrose synthase domain were missing and the motif composition of some members were found to be variants in apple, pear, peach, and soybean (Fig. 2B). The five PgSUS members also shared common conserved motif compositions and had consistent arrangement (motifs 12, 9, 10, 11, 6, 3, 13, 1, 5, 7, 4, 2, 8 and 15) (Fig. 2B).

Tow typically conserved domains corresponding to the motifs features (sucrose synthase domain and the glycosyl-transferase domain) were screened in each member of 68 SUS proteins by matching with SMART and NCBI CDD (Fig. 2C). These two conserved domains were located at the $\mathrm{N}$ and $\mathrm{C}$-terminal ends, respectively. This was consistent with the motif arrangement (Fig. 2B,C). In pomegranate, the length and distribution of two conserved domains of five SUS protein showed high consistency and conservation (Fig. 2C), indicating that they are critical for the function of PgSUS proteins.

\section{Prediction of protein structure of pomegranate SUS proteins}

The secondary structures analysis showed that five pomegranate SUS proteins were composed of $\alpha$-helices, extended $\beta$ strands, $\beta$-turns, and random coils (Table 2; Data S9). The $\alpha$-helix was the major secondary structures among the five PgSUS proteins, accounting for $49.72-53.97 \%$, followed by random coils (25.73-32.74\%) and extended $\beta$ strands (12.26-13.21\%) (Table 2). These secondary structure distributions were also highly conserved in five PgSUS polypeptide chains (Data S9).

We predicted tertiary structures of the five pomegranate SUS proteins using the Swiss-model online software. The three-dimensional models of the PgSUSs proteins were based on templates 3s27 (Sucrose synthase) and 4rbn (Glycosyl transferases group 1). The results showed that the tertiary structure for PgSUS1 to PgSUS5 had two symmetric tetramers and comprised four main polypeptide chains. These were similar to PpSus1 to PpSus4 in peach (Data S10; Zhang et al., 
294 2015). The 3D structure of PgSUS1 was quite similar with PgSUS4 among PgSUS1 to PgSUS5 295 (Data S10).

\section{Syntenic analysis of five species $\boldsymbol{S} U \boldsymbol{S}$ genes}

297 We analyzed the syntenic relationships between pomegranate and four other species, including

298 A. thaliana, M. domestica, . bretschneideri, and $V$. vinifera to explore the evolutionary process

299 of pomegranate $S U S$ genes. Four SUS genes were found to have ten orthologous syntenic gene

300 pairs in another four species (Fig. 3). PgSUS1 was found to be syntenic with four genes from

301 apple (MdSUS1.1 and MdSUS1.4), pear (PbrSUS17), and grape (VvSS4). Three genes (AtSUS6,

302 MdSUS3.1 and PbrSUS12) showed synteny with PgSUS5, two genes (PbrSUS17 and VvSS4)

303 were syntenic with $P g S U S 4$, and $P g S U S 3$ was syntenic only with $V v S S 3$ (Fig. 3). The syntenic

304 relationships of these $S U S$ orthologous gene pairs were consistent with their phylogenetic

305 relationship (Fig. 1). However, PgSUS2 in pomegranate was found to have no syntenic

306 counterpart in the other four species. These results help to better understanding the possible roles

307 of SUS gene family members in pomegranate.

\section{Cis-acting element analysis of $\boldsymbol{P g S U S}$ genes promoters}

309 The cis-acting elements are crucial in the spatial-temporal and tissue-specific expression of

310 genes. The cis-acting elements of the PgSUS genes were classified into five categories using

311 the PlantCARE database The categories were: hormone responsive elements (HRE), tissue

312 specific elements (TSE), light responsive elements (LRE), stress responsive elements (SRE), and

313 others responsive elements (ORE) (Fig. 4). Detailed information of cis-acting elements in five

$314 P g S U S$ promoter regions is provided in Data S11. The number of LREs was the largest group

315 (49\%), followed by HREs (24\%), SREs (15\%), OREs (7\%) and TSEs (6\%) (Fig. 4A; Data S11).

316 Among these, the presence of LREs was universal in five PgSUS genes promoters. The PgSUS3

317 promoter contained 22 LREs, which was almost two times that of other PgSUS genes These

318 results imply that $P g S U S 3$ may respond to light induction. Other $P g S U S$ genes promoters

319 contained several HREs, with the exception of PgSUS2. These hormones include abscisic acid

320 (ABA), auxin, gibberellin (GA), methyl jasmonate (MeJA), and salicylic acid (SA). MeJA and

321 ABA responsive elements were prevalent in the promoter regions of those four genes. Moreover,

322 each PgSUS promoter contained tow-to-eight SREs, and were responsive to stresses including

323 anoxic environments, low-temperatures, and drought (Fig. 4B; Data S11). In addition, PgSUS

324 genes promoters also contained several OREs, such as circadian control, cell cycle regulation,

325 and MYB binding sites, implying that PgSUS family genes may play multiple roles in plant

326 growth and development.

327 Expression profile of pomegranate $\boldsymbol{S} \boldsymbol{U} \boldsymbol{S}$ family genes, assessed with RNA-seq and qPCR

328 In order to analyze the molecular functions of the SUS genes in pomegranate, we studied the

329 transcript characteristics of the PgSUS genes using RNA-seq data downloaded from the NCBI 
330 SRA database (Fig. 5). For transcriptome analysis, a total of $300.88 \mathrm{~Gb}$ clean data with an 331 average of $94.49 \%$ bases scoring Q30 were obtained from 42 RNA-seq libraries. The GC content 332 of all samples ranged from 49.50 to $52.80 \%$. It was found that more than $96 \%$ of the reads 333 aligned with the pomegranate genome sequence, indicating a high sequencing quality and that 334 the resulting data was reliable for subsequent analyses (Data S12).

PgSUS genes exhibited an obvious tissue specific expression pattern (Fig. 5A). The PgSUS family members of the SUS I and SUS II subgroups were predominantly expressed in sink organs, particularly in fruit tissues. With the fruit development, PgSUS1, PgSUS3, and PgSUS4 transcripts displayed different expression characteristics. PgSUS1 was mainly expressed in the inner and outer seed coats, and reached its peak at 95 DAF in the outer seed coat. The $P g S U S 3$ transcript was expressed at higher levels in the seed coat and pericarp (Fig. 5A). As the pericarp developed from 50 DAF to 95 DAF, PgSUS3 expression gradually increased to the highest level, but slightly declined at fruit harvest (Fig. 5A). Interestingly, PgSUS4 was strongly expressed in the sink organ, including the outer seed coat, root, and flower. PgSUS4 showed a similar expression trend with PgSUS1 as the outer seed coat developed from 50 DAF to 140 DAF. Its abundance rapidly increased on the 95 DAF (Fig. 5A). However, PgSUS2 and PgSUS5 of the SUS III subgroup were slightly expressed in the root, leaf, and flower, but was almost undetectable in fruits tissues (Fig. 5A). Furthermore, similar expression trends of PgSUS genes were also observed during the fruit development in the 'Dabenzi' and 'Tunisia' pomegranate cultivars (Fig. 5B).

QPCR was used to analyze the expression patterns of $P g S U S$ genes. The relative expression level of each gene in different organs or tissues was standardized with their expression level in the leaf (Fig. 6). All five genes were up-regulated in root and flower compared with their expressions in the leaf. The relative expression level of PgSUS4 increased more significantly than the other PgSUSs genes in root and flower. During fruit development, expressions of PgSUS1 and PgSUS4 rapidly increased with a tendency toward to a gradual decrease as the seed coat developed from $45 \mathrm{DAF}$ to $150 \mathrm{DAF}$, which peaked at $75 \mathrm{DAF}$. These results suggest that isozymes encoded by these two genes may be involved in catalyzing key aspects of sucrose metabolism in the fruit seed coat during the early- and middle- developmental stages. PgSUS3 showed stable expression levels when the fruit seed coat developed from 45 DAF to 115 DAF. Additionally, $P g S U S 3$ showed higher transcript levels in the pericarp than other genes, indicating that PgSUS3 may play an important role in sucrose metabolism during the development of the pomegranate fruit pericarp. However, the transcripts levels of PgSUS2 and PgSUS5 were slightly or not-at-all expressed during fruit development. Our results show that three $S U S$ genes (PgSUS1, PgSUS3 and PgSUS4) may contribute to the sucrose metabolism and fruit development. 


\section{Discussion}

367 Sucrose is synthesized in the leaf and transported to sink tissues, where it participates in growth and development, carbohydrate consumption, or the synthesis of major storage products in sink organs. In pomegranate, several key enzymes or genes play roles in sucrose metabolism in multiple sink organ or tissues, such as vegetative shoot apices, unpollinated ovaries, and seed (Meletis et al., 2019; Poudel et al., 2020). However, the molecular function of the SUS gene family as one of the key genes of sucrose metabolism in pomegranate remains unknown. Recently, more members of the SUS gene family have been identified and characterized from different plant species using comparative genome approaches and research advances in plant whole-genome sequencing, assembly, and annotation (Stein \& Granot, 2019). The number of SUS family members differed among plant species, however, the SUS family typically contained four to seven genes (Stein \& Granot, 2019). We identified at least five SUS genes in the pomegranate genome belonging to typical genes in the SUS family. (Table 1). The average length of the SUS polypeptide chain was approximately 800 amino acids and the monomers weight was approximately $90 \mathrm{kDa}$ (Stein \& Granot, 2019), such as in citrus CitSUS 1-6 (Islam et al., 2019), peach PpSUS1, 2, and 5 (Zhang et al., 2015), grape VvSS1-4 (Zhu et al., 2017), and pomegranate PgSUS1-4 (Table 1). The monomer weight of several other SUS isoforms was different with the members mentioned above. For example, the Arabidopsis AtSUS6 monomer weighs about $107 \mathrm{kDa}$ (Baud et al., 2004), and grape VvSS5 was estimated to be $102.7 \mathrm{kDa}$ (Zhu et al., 2017). The weight of peach PpSUS6 (Zhang et al., 2015), Indian mustard BjSUS6.1, 6.2, 7.1, and 7.2 (Koramutla et al., 2019), and pomegranate PgSUS5 were estimated to be above100 $\mathrm{kDa}$. Most of the pomegranate SUS proteins were predicted to be hydrophilic, with a low instability index, and contained acidic amino acids (Table 1), which was similar to the physical and chemical properties of other plant SUS proteins (Islam et al., 2014; Tong et al., 2018). Moreover, two putative Ser phosphorylation sites were observed in the N-terminal regions of all PgSUS proteins (Data S3), which may help determine the SUS subcellular localization and enzyme activity (Stein \& Granot, 2019). Pomegranate SUS genes also shared a high sequence similarity of CDS and amino acid with 63 other SUS genes (Data S4). Therefore, the predicted molecular physicochemical characteristics of the five pomegranate SUS proteins were similar to be SUS proteins identified previously in other plant species.

The results of phylogenetic tree helped to predict the possible origin and relationships among different SUS isoforms. Although the SUS family genes shared high sequence similarities (Data S4), phylogenetic result indicated that diversification occurred in this family. The SUS family has been historically classified into three major subfamilies in plants, namely SUS I, SUS II, and SUS III (Xu et al., 2014). The phylogenetic results of this study supported that the five PgSUS 
401

402

403

404

405

406

407

408

409

410

411

412

413

414

415

416

417

418

419

420

421

422

423

424

425

426

427

428

429

430

431

432

433

434

435

436

candidates were also categorized into distinct subgroups together with other SUS orthologs in Arabidopsis (Baud et al., 2004), apple (Tong et al., 2018), and other species (Stein \& Granot, 2019) (Fig. 1). SUS I was further classified into monocot- and dicot-specific subgroups (Chen et al., 2012; Koramutla et al., 2019; Xu et al., 2019). In pomegranate, PgSUSI and PgSUS4 were clustered together with 17 other SUS genes of dicots into SUS I (Fig. 1), suggesting that a gene duplication event resulting in pomegranate $P g S U S 1$ and PgSUS4 may have occurred after the split of monocots and dicots (Chen et al., 2012; Koramutla et al., 2019; Xu et al., 2019).

Moreover, since PgSUS1 and PgSUS4 were grouped closely together and formed an independent pomegranate clade separate from Arabidopsis, pear, apple, peach, and other dicots genes. The independent gene duplication may have given rise to PgSUS1 and PgSUS4, which may have occurred more recently after pomegranate's separation from Arabidopsis and Rosaceae species. The generation of the PgSUS2 and PgSUS5 genes, clustered together into SUS III, may have taken place before the separation of Punicaceae/Arabidopsis. We also observed the C-terminal extension in pomegranate SUS III subfamily genes (Data S5), implying that SUS III genes may derive from a common ancestor, which was consistent with previous studies (Xu et al., 2014). Additionally, PgSUS3 and other members from both dicot and monocot species were grouped together into SUS II. These results support the view that SUS II and III subgroups are evolutionarily older than SUS I dicot subgroup (Zhu et al., 2017; Chen et al., 2012; Koramutla et al., 2019).

Intron and exon structures provide valuable information for the discovery of gene phylogenies (Lecharny et al., 2003). The intron loss event during ancient SUS genes evolution was proposed to be a common phenomenon, especially in the SUS I and SUS III gene subgroups ( $X u$ et al., 2014). For instance, some introns may have been lost in PgSUS1, PgSUS4, PgSUS2, and $P g S U S 5$. Intriguingly, the exon/intron structures of PgSUS3 showed greater similarity to the putative ancestral genes of the SUS II subgroups (Data S6; Data S7), in which intron loss events occurred seldomly (Xu et al., 2014). These results support the hypothesis that the SUS II subgroup likely possessed relatively lower evolutionary rates (Chen et al., 2012; Wang et al., 2015; Koramutla et al., 2019; Xu et al., 2019). The additional exons in the 3' end of PgSUS2 and PgSUS5 of SUS III were similar to the amino acid alignment (Data S5), leading to the complexity of intron/exon structure (Data S7). However, the function of the 3' extension was unclear (Xu et al., 2014). Therefore, the evolutionary and functional effects of intron loss as well as the $3^{\prime}$ extension in the SUS genes requires additional research.

The motif composition and arrangement determinate the signature of the protein domain. SUS proteins showed conserved structural motifs among different plant species (Zhang et al., 2015; Koramutla et al., 2019). The motifs of five PgSUSs in this study shared extremely high similarities, suggesting that the pomegranate $S U S$ genes were more conserved during evolution. 
437 Two common typical domains of SUS proteins were identified in several family members based

438

439 on the similarity of nucleotide and peptide chain sequences, conserved exons, and motif arrangements (Zhang et al., 2015; Koramutla et al., 2019), including five PgSUS proteins (Fig. $2 \mathrm{C}$ ), which confirmed their authenticity in the pomegranate genome. The secondary and tertiary structure prediction of proteins provided the opportunity to obtain insights into understanding its biological functions (Krissinel et al., 2004). Differences in the physicochemical characteristics of the protein sequences of five PgSUS genes resulted in their protein being folded into specific two- and three-dimensional structures (Data S9-10). Among five pomegranate SUS proteins, the 2-D and 3-D of PgSUS1 and PgSUS4 were very similar, which was consistent with their close evolutionary relationship (Fig. 1) and implies that they may share similar functions. These results suggest that $P g S U S$ family genes were highly conserved during evolution, despite the small differences found.

Whole-genome duplication (WGD), segmental duplication, and tandem duplication are the common gene duplication events in plants, which facilitated to gene family expansion and functional diversification (Flagel et al., 2009). Although segmental or tandem duplication was suggested as the predominant pattern for the expansion of SUS family in pear (Abdullah et al., 2018), these types were not detected in PgSUS genes. This may explain the presence of relatively fewer SUS family members in pomegranate (Fig. 3; Table 1). In addition, four PgSUSs genes showed syntenic relationships with the genes of the other four species (Fig. 3), confirming their closer phylogenetic relationship, and their functional similarities.

In the gene promoter region, cis-acting elements may bind with specific transcription factors to modulate transcriptional levels, and respond to the stimulate signal (Riechmann \& Ratcliffe, 2000). Light is an important environmental factor that may affect the storage or breakdown of sugars in roots, stems, and fruits in some biological metabolism, which then requires a series of enzymes, including sucrose synthase (Girault et al., 2010). In wheat, light illumination upregulated the SUS2 mRNA level, but decreased SUS1 expression (Marana et al., 1990). Compared with full-sun conditions, a higher expression of CaSUS2 led to the improved hydrolytic activity of sucrose synthase in mature endosperm of coffee fruits under shade (Geromel et al., 2008). Here, the promoter prediction indicated that LREs occupied a larger proportion in the promoter region (Fig. 4A; Data S11), which was previously observed in Indian mustard and pear (Koramutla et al., 2019; Abdullah et al., 2018) These results indicate that light may be an important factor in the transcript regulation of PgSUSs genes. Moreover, research suggests that phytohormones may regulate the SUS expression level. In rice, SUS expression was induced by ABA during grain filling (Tang et al., 2009). In cotton, GAs promoted GhSUSA1 expression, which resulted in the secondary cell wall deposition of fibers (Bai et al., 2014). The SUS gene was involved in the auxin-signaling pathway in tomato (Goren et al., 2017). Therefore, 
473

474

475

476

477

478

479

480

481

482

483

484

485

486

487

488

489

490

491

492

493

494

495

496

497

498

499

500

501

502

503

504

505

506

507

508

the presence of HREs predicted in the promoter region of pomegranate SUS genes implied their capacity to respond to phytohormones (Fig. 4; Data S11). SUS expression was also associated withstressors, such as low-oxygen, cold, heat, salinity, and drought (Wang et al., 2015; Stein \& Granot 2019; Zhu et al., 2017). SREs were found to be universally distributed in each PgSUS promoter, indicating that $P g S U S$ genes may respond to abiotic stresses in pomegranate (Fig. 4B; Data S11). Therefore, predictive promoter analysis facilitates our understanding of the multiple functions of $P g S U S$ genes during pomegranate growth and development.

Several studies have shown that $S U S$ genes exhibit tissue-specific and development-dependent expression profiles, primarily in the sink organs. AtSUS2 was specifically induced in seeds (Baud et al., 2004). The expression level of ZmSUS3 gradually increased during the maize kernel maturation process (Carlson et al., 2002). The poplar PtSUS genes showed high transcript levels in roots, vegetative buds, and floral catkins (An et al., 2014). VvSS1 expression in grape reached its peak at the start of young leaf development (Zhu et al., 2017). Likewise, the transcription and qPCR data presented in this study suggested the significant expression of some PgSUS genes (PgSUS1, PgSUS3 and PgSUS4) in pomegranate sink organs (Fig. 5,6). However, the expression levels of PgSUS2 and PgSUS5 were at low levels or undetected, indicating they might be redundant for pomegranate during the normal growth and development process. In edible fruits, the most important sink organ is fruit. SUS shows its close relationship with fruit development in several horticultural plants. For instance, CitSUS1, CitSUS2 of the SUS I subgroup and CitSUS6 of the SUS II subgroup were notably expressed in the juice sacs of citrus fruit (Islam et al., 2019). In peach, PpSUS1 of the SUS I reached its highest levels during fruit maturation, while PpSUS5 of SUS III was predominantly expressed in the early stages of fruit development (Zhang et al., 2015). PbrSUS2 and PbrSUS15 of SUS I were significantly up-regulated in pear fruit ( $\mathrm{Lv}$ et al., 2018). MdSUS1.1/1.2/1.4 of SUS I and MdSUS2.1 of SUS II were mainly expressed in young and mature apple fruits, respectively (Tong et al., 2018). In this study, the significant expression of PgSUS1, PgSUS4, and PgSUS3 were detected in different fruit tissues (Fig. 5,6). The expression on PgSUS1 in SUS I was quite high in early and mid-development stages of the fruit seed coat, which is the main edible part of the pomegranate. These results are consistent with the MdSUS1.1 expression pattern, and confirms their evolutionary and syntenic relationships (Fig. 1,3,5,6). PgSUS3 and PgSUS4 were also highly expressed in the seed coat, with differential but partially overlapping expression patterns. Therefore, PgSUS1 and PgSUS4 of SUS I and PgSUS3 of SUS II may play important regulatory roles in sucrose metabolism in the seed coat, as well as the quality of the fruit. These results also confirmed the molecular function of several members clustered into the SUS II subgroup that may overlap with the SUS I genes in specific plants (Xu et al., 2014). PgSUS3 expression was notably increased in the pericarp, implying that $P g S U S 3$ could be closely related with the sucrose metabolism of the fruit 
509

510

511

512

513

514

515

516

517

518

519

520

521

522

523

524

525

526

527

528

529

530

531

532

533

534

535

536

537

538

539

540

541

542

543

544

545

pericarp PgSUS4 was highly expressed both the root and flower, suggesting that PgSUS4 may specially regulate sucrose metabolism in these sink organs with the exception of its functional redundancy in fruit development. These results imply that SUS genes in pomegranate may play crucial roles in pomegranate sucrose metabolism, particularly in fruit development.

\section{Conclusions}

Our results show that the five sucrose synthase genes identified in the pomegranate genome, were clustered into three distinct subgroups. The structures of different SUS genes in pomegranate were highly conserved during evolution and they might play different roles in sucrose metabolism and fruit development due to their partially overlapping but distinctly variable expression patterns. Our results further the understanding of the molecular basis of sucrose synthase genes in pomegranate. Future studies, including the analysis of gene overexpression and suppression, are needed to determine the specific functions of PgSUS1, PgSUS3, and PgSUS4 in fruit sugar metabolism.

\section{References}

Abdullah M, Cao Y, Cheng X, Meng D, Chen Y, Shakoor A, Gao J, Cai Y. 2018. The Sucrose Synthase Gene Family in Chinese Pear (Pyrus bretschneideri Rehd.): Structure, Expression, and Evolution. Molecules 23(5): 1144. Doi 10.3390/molecules23051144.

An X, Chen Z, Wang J, Ye M, Ji L, Wang J, Liao W, Ma H. 2014. Identification and characterization of the Populus sucrose synthase gene family. Gene 539(1): 58-67. Doi 10.1016/j.gene.2014.01.062.

Bai WQ, Xiao YH, Zhao J, Song SQ, Hu L, Zeng JY, Li XB, Hou L, Luo M, Li DM, Pei Y. 2014. Gibberellin overproduction promotes sucrose synthase expression and secondary cell wall deposition in cotton fibers. PLoS One 9(5): e96537. Doi 10.1371/journal.pone.0096537.

Baud S, Vaultier MN, Rochat C. 2004. Structure and expression profile of the sucrose synthase multigene family in Arabidopsis. J. Exp. Bot 55(396): 397-409. Doi 10.1093/jxb/erh047.

Blom N, Sicheritz-Pontén T, Gupta R, Gammeltoft S, Brunak S. 2004. Prediction of post-translational glycosylation and phosphorylation of proteins from the amino acid sequence. Proteomics 4(6): 1633-1649. Doi 10.1002/pmic.200300771.

Bolger AM, Lohse M, Usadel B. 2014. Trimmomatic: a flexible trimmer for Illumina sequence data. Bioinformatics 30(15): 2114-2120. Doi 10.1093/bioinformatics/btu170.

Carlson SJ, Chourey PS, Helentjaris T, Datta R. 2002. Gene expression studies on developing kernels of maize sucrose synthase (SuSy) mutants show evidence for a third SuSy gene. Plant Mol Biol 49(1): 15-29. Doi 10.1023/a:1014457901992.

Chen A, He S, Li F, Li Z, Ding M, Liu Q, Rong J. 2012. Analyses of the sucrose synthase gene family in cotton: structure, phylogeny and expression patterns. BMC plant biol 12: 85. Doi 10.1186/1471-2229-12-85.

Chen C, Chen H, Zhang Y, Thomas HR, Frank MH, He Y, Xia R. 2020. TBtools: An Integrative Toolkit Developed for Interactive Analyses of Big Biological Data. Mol plant 13(8): 1194-1202. Doi 10.1016/j.molp.2020.06.009.

Chourey PS, Taliercio EW, Carlson SJ, Ruan YL. 1998. Genetic evidence that the two isozymes of sucrose synthase present in developing maize endosperm are critical, one for cell wall integrity and the other for starch biosynthesis. Mol Gen Genet 259(1): 88-96. Doi 10.1007/s004380050792.

Peer] reviewing PDF | (2021:08:64364:1:0:NEW 5 Dec 2021) 
Conidi C, Drioli E, Cassano A. 2020. Perspective of membrane technology in pomegranate juice processing: A Review. Foods 9(7): E889. Doi 10.3390/foods9070889.

Craig J, Barratt P, Tatge H, Déjardin A, Handley L, Gardner CD, Barber L, Wang T, Hedley C, Martin C, Smith AM. 1999. Mutations at the rug4 locus alter the carbon and nitrogen metabolism of pea plants through an effect on sucrose synthase. Plant J 17: 353-62. Doi 10.1046/j.1365-313X.1999.00382.x.

D'Aoust MA, Yelle S, Nguyen-Quoc B. 1999. Antisense inhibition of tomato fruit sucrose synthase decreases fruit setting and the sucrose unloading capacity of young fruit. Plant Cell 11(12): 2407-2418. Doi 10.1105/tpc.11.12.2407.

Dominguez PG, Donev E, Derba-Maceluch M, Bünder A, Hederström M, Tomáŝková I, Mellerowicz EJ, Niittylä T. 2021. Sucrose synthase determines carbon allocation in developing wood and alters carbon flow at the whole tree level in aspen. New Phyto 229: 186-198. Doi 10.1111/nph.16721.

Duan Y, Yang L, Zhu H, Zhou J, Sun H, Gong H. 2021. Structure and expression analysis of sucrose phosphate synthase, sucrose synthase and invertase gene families in Solanum lycopersicum. Int J Mol Sc 22(9): 4698. Doi:10.3390/ijms22094698.

Duncan KA, Hardin SC, Huber SC. 2006. The three maize sucrose synthase isoforms differ in distribution, localization, and phosphorylation. Plant Cell Physiol 47(7): 959-971. Doi 10.1093/pcp/pcj068.

Finn RD, Clements J, Arndt W, Miller BL, Wheeler TJ, Schreiber F, Bateman A, Eddy SR. 2015. HMMER web server: 2015 update. Nucleic Acids Res 43(W1): W30-W38. Doi 10.1093/nar/gkv397.

Flagel LE, Wendel JF. 2009. Gene duplication and evolutionary novelty in plants. New Phytol 183(3): 557-564. Doi 10.1111/j.1469-8137.2009.02923.x.

Geromel C, Ferreira LP, Davrieux F, Guyot B, Ribeyre F, Brígida dos Santos Scholz M, Protasio Pereira LF, Vaast P, Pot D, Leroy T, Androcioli Filho A, Esteves Vieira LG, Mazzafera P, Marraccini P. 2008. Effects of shade on the development and sugar metabolism of coffee (Coffea arabica L.) fruits. Plant Physiol Biochem 46(5-6): 569-579. Doi 10.1016/j.plaphy.2008.02.006.

Girault T, Abidi F, Sigogne M, Pelleschi-Travier S, Boumaza R, Sakr S, Leduc N. 2010. Sugars are under light control during bud burst in Rosa sp. Plant Cell Environ 33(8): 1339-1350. Doi 10.1111/j.1365-3040.2010.02152.x.

Goren S, Lugassi N, Stein O, Yeselson Y, Schaffer AA, David-Schwartz R, Granot D. 2017. Suppression of sucrose synthase affects auxin signaling and leaf morphology in tomato. PloS one 12(8): e0182334. Doi 10.1371/journal.pone.0182334.

Hirose T, Scofield G, Terao T. 2008. An expression analysis profile for the entire sucrose synthase gene family in rice. Plant Sci 174(5): 534-543. Doi 10.1016/j.plantsci.2008.02.009.

Islam MZ, Hu XM, Jin LF, Liu YZ, Peng SA. 2014. Genome-wide identification and expression profile analysis of citrus sucrose synthase genes: investigation of possible roles in the regulation of sugar accumulation. PloS one 9(11): e113623. Doi 10.1371/journal.pone.0113623.

Kim D, Paggi JM, Park C, Bennett C, Salzberg SL. 2019. Graph-based genome alignment and genotyping with HISAT2 and HISAT-genotype. Nat Biotechnol 37(8): 907-915. Doi 10.1038/s41587-019-0201-4.

Koramutla MK, Ram C. Bhatt D, Annamalai M, Bhattacharya R. 2019. Genome-wide identification and expression analysis of sucrose synthase genes in allotetraploid Brassica juncea. Gene 707: 126-135. Doi 10.1016/j.gene.2019.04.059.

Krissinel E, Henrick K. 2004. Secondary-structure matching (SSM), a new tool for fast protein structure alignment in three dimensions. Acta Crystallogr D Biol Crystallogr 60(Pt 12 Pt 1): 2256-2268. Doi 10.1107/S0907444904026460.

Lecharny A, Boudet N, Gy I, Aubourg S, Kreis M. 2003. Introns in, introns out in plant gene families: a genomic approach of the dynamics of gene structure. J Struct Funct Genom 3(1-4): 111-116.

Letunic I, Bork P. 2018. 20 years of the SMART protein domain annotation resource. Nucl Acids Res 46(D1): D493-D496. Doi 


\subsection{3/nar/gkx922.}

Li F, Hao C, Yan L, Wu B, Qin X, Lai J, Song Y. 2015. Gene structure phylogeny and expression profile of the sucrose synthase gene family in cacao (Theobroma cacao L.). J Genet 94(3): 461-472. Doi 10.1007/s12041-015-0558-1.

Li J, Gao K, Lei B, Zhou J, Guo T, An X. 2020. Altered sucrose metabolism and plant growth in transgenic Populus tomentosa with altered sucrose synthase PtSS3. Transgenic Res 29(1): 125-134. Doi 10.1007/s11248-019-00184-9.

Li M, Feng F, Cheng L. 2012. Expression patterns of genes involved in sugar metabolism and accumulation during apple fruit development. PLoS One 7(3): e33055. Doi 10.1371/journal.pone.0033055.

Li M, Wang S, Liu Y, Zhang Y, Ren M, Liu L, Lu T, Wei H, Wei Z. 2019. Overexpression of PsnSuSyl, 2 genes enhance secondary cell wall thickening, vegetative growth, and mechanical strength in transgenic tobacco. Plant Mol Biol 100(3): 215-230. Doi 10.1007/s11103-019-00850-w.

Qin G, Liu C, Li J, Qi Y, Gao Z, Zhang X, Yi X, Pan H, Ming R, Xu Y. 2020. Diversity of metabolite accumulation patterns in inner and outer seed coats of pomegranate: exploring their relationship with genetic mechanisms of seed coat development. Horti Res 7:10. DOI 10.1038/s41438-019-0233-4.

Livak KJ, Schmitten TD. 2001. Analysis of relative gene expression data using real-time quantitative PCR and the $2^{-\Delta \Delta C T}$ method. Methods 25(4): 402-408. Doi 10.1006/meth.2001.1262.

Lu S, Wang J, Chitsaz F, Derbyshire MK, Geer RC, Gonzales NR, Gwadz M, Hurwitz DI, Marchler GH, Song JS, Thanki N, Yamashita RA, Yang M, Zhang D, Zheng C, Lanczycki CJ, Marchler-Bauer A. 2020. CDD/SPARCLE: the conserved domain database in 2020. Nucleic Acids Res 48(D1): D265-D268. Doi 10.1093/nar/gkz991.

Luo X, Li H, Wu Z, Yao W, Zhao P, Cao D, Yu H, Li K, Poudel K, Zhao D, Zhang F, Xia X, Chen L, Wang Q, Jing D, Cao S. 2020. The pomegranate (Punica granatum L.) draft genome dissects genetic divergence between soft- and hardseeded cultivars. Plant Biotechnol J 18(4): 955-968. Doi 10.1111/pbi.13260.

Lutfiyya L, Xu N, D’Ordine R, Morrell J, Miller P, Duff S. 2007. Phylogenetic and expression analysis of sucrose phosphate synthase isozymes in plants. J Plant Physiol 164(7): 923-933. Doi 10.1016/j.jplph.2006.04.014.

Lv JH, Wang YZ, Cheng R, Wang GM, Zhang SL, Wu J, Zhang HP. 2018. Genome-wide identification and expression analysis of Sucrose Synthase (SUS) and Sucrose Phosphate Synthase (SPS) gene families in pear. Acta Hortic Sin 45(3): 421-435. Doi 10.16420/j.issn.0513-353x.2017-0474.

Maraña C, García-Olmedo F, Carbonero P. 1990. Differential expression of two types of sucrose synthase-encoding genes in wheat in response to anaerobiosis cold shock and light. Gene 88(2): 167-172. Doi 10.1016/0378-1119(90)90028-p.

Meletis K, Tsaniklidis G, Papadakis IE, Vemmos SN. 2019. Sucrose synthesis in Unpollinated ovaries of pomegranate (Punica granatum L.), as well as, in reproductive and vegetative shoot apices. Biologia 74, 111-117. Doi 10.2478/s11756018-0154-y.

Pertea M, Pertea GM, Antonescu CM, Chang TC, Mendell JT, Salzberg SL. 2015. StringTie enables improved reconstruction of a transcriptome from RNA-seq reads. Nat Biotechnol 33(3): 290-295. Doi 10.1038/nbt.3122.

Poudel K, Luo X, Chen L, Jing D, Xia X, Tang L, Li H, Cao S. 2020. Identification of the $S U T$ gene family in pomegranate (Punica granatum L.) and functional analysis of PgL0145810.1. Int J Mol Sci 21(18): 6608. Doi 10.3390/ijms21186608.

Qin G, Xu C, Ming R, Tang H, Guyot R, Kramer E, M, Hu Y, Yi X, Qi Y, Xu X, Gao Z, Pan H, Jian J, Tian Y, Yue Z, Xu Y. 2017. The pomegranate (Punica granatum L.) genome and the genomics of punicalagin biosynthesis. Plant J 91(6): 1108-1128. Doi 10.1111/tpj.13625.

Riechmann JL, Ratcliffe OJ. 2000. A genomic perspective on plant transcription factors. Curr Opin Plant Biol 3(5): $423-434$. Doi 10.1016/s1369-5266(00)00107-2.

Stein O, Granot D. 2019. An Overview of Sucrose Synthases in Plants. Front Plant Sci 10: 95. Doi 10.3389/fpls.2019.00095. 
Tang T, Xie H, Wang Y, Lü B, Liang J. 2009. The effect of sucrose and abscisic acid interaction on sucrose synthase and its relationship to grain filling of rice (Oryza sativa L.). J Exp Bot 60(9): 2641-2652. Doi 10.1093/jxb/erp114.

Tong, XL, Wang ZY, Ma BQ, Zhang CX, Zhu LC, Ma FW, Li MJ. 2018. Structure and expression analysis of the sucrose synthase gene family in apple. J Integra Agri 17(4): 847-856. Doi 10.1016/S2095-3119(17)61755-6.

Wang Y, Tang H, Debarry JD, Tan X, Li J, Wang X, Lee TH, Jin H, Marler B, Guo H, Kissinger JC, Paterson AH. 2012. MCScanX: a toolkit for detection and evolutionary analysis of gene synteny and collinearity. Nucleic Acids Res 40(7): e49. Doi 10.1093/nar/gkr1293.

Wang Z, Wei P, Wu M, Xu Y, Li F, Luo Z, Zhang J, Chen A, Xie X, Cao P, Lin F, Yang J. 2015. Analysis of the sucrose synthase gene family in tobacco: structure phylogeny and expression patterns. Planta 242(1): 153-166. Doi 10.1007/s00425015-2297-1.

Wei Z, Qu Z, Zhang L, Zhao S, Bi Z, Ji X, Wang X, Wei H. 2015. Overexpression of poplar xylem sucrose synthase in tobacco leads to a thickened cell wall and increased height. PloS one 10(3): e0120669. Doi 10.1371/journal.pone.0120669.

Xu F, Joshi C. 2010. Overexpression of aspen sucrose synthase gene promotes growth and development of transgenic Arabidopsis plants. Adv Biosci Biotechnol 1(05): 426-438. Doi 10.4236/abb.2010.15056.

Xu X, Yang Y, Liu C, Sun Y, Zhang T, Hou M, Huang S, Yuan H. 2019. The evolutionary history of the sucrose synthase gene family in higher plants. BMC plant biol 19(1): 566. Doi 10.1186/s12870-019-2181-4.

Yuan Z, Fang Y, Zhang T, Fei Z, Han F, Liu C, Liu M, Xiao W, Zhang W, Wu S, Zhang M, Ju Y, Xu H, Dai H, Liu Y, Chen Y, Wang L, Zhou J, Guan D, Yan M, Xia Y, Huang X, Liu D, Wei H, Zheng H. 2018. The pomegranate (Punica granatum L.) genome provides insights into fruit quality and ovule developmental biology. Plant Biotechnol J 16(7): 13631374. Doi 10.1111/pbi.12875.

Zhang C, Yu M, Ma Z, Shen Z, Zhang B, Korir KN. 2015. Structure expression profile and evolution of the sucrose synthase gene family in peach (Prunus persica). Acta Physiol Plant 37: 81. Doi 10.1007/s11738-015-1829-4.

Zhao C, Hua LN, Liu XF, Li YZ, Shen YY, Guo JX. 2017. Sucrose synthase FaSS1 plays an important role in the regulation of strawberry fruit ripening. Plant Growth Regul 81: 175-181. Doi 10.1007/s10725-016-0189-4.

Zhu X, Wang M, Li X, Jiu S, Wang C, Fang J. 2017. Genome-Wide Analysis of the Sucrose Synthase Gene Family in Grape (Vitis vinifera): Structure Evolution and Expression Profiles. Genes 8(4): 111. Doi 10.3390/genes8040111. 


\section{Table $\mathbf{1}$ (on next page)}

Table 1. The characteristics of the sucrose synthase genes in pomegranate

CDS, Coding DNA sequence; MW, molecular weight; pl, isoelectric point; Ai, aliphatic index; GRAVY, grand average of hydropathicity. 
1 Table 1. The characteristics of the sucrose synthase genes in pomegranate

\begin{tabular}{|c|c|c|c|c|c|c|c|c|c|c|}
\hline $\begin{array}{l}\text { Gene } \\
\text { name }\end{array}$ & Gene ID & $\begin{array}{l}\text { Genome } \\
\text { location }\end{array}$ & $\begin{array}{c}\text { cDNA } \\
\text { length } \\
\text { (bp) }\end{array}$ & $\begin{array}{c}\text { CDS } \\
\text { length } \\
\text { (bp) }\end{array}$ & $\begin{array}{c}\text { Protein } \\
\text { length } \\
\text { (aa) }\end{array}$ & $\begin{array}{c}\text { MW } \\
\text { (KDa) }\end{array}$ & $\begin{array}{l}\text { Theoretical } \\
\text { pI index }\end{array}$ & $\begin{array}{l}\text { Instability } \\
\text { index }\end{array}$ & $\mathbf{A i}$ & GRAVY \\
\hline PgSUS1 & $\begin{array}{l}\text { XM_031 } \\
527544.1\end{array}$ & $\begin{array}{c}\text { Chr02:16303127 } \\
\ldots 16309696(-)\end{array}$ & 6570 & 2421 & 806 & 92.78 & 6.56 & 33.79 & 92.25 & -0.262 \\
\hline PgSUS2 & $\begin{array}{l}\text { XM_031 } \\
535599.1\end{array}$ & $\begin{array}{c}\text { Chr04:6084363 } \\
\ldots 6088611(+)\end{array}$ & 4249 & 2499 & 832 & 94.97 & 5.99 & 36.58 & 81.60 & -0.397 \\
\hline PgSUS3 & $\begin{array}{l}\text { XM_031 } \\
544401.1\end{array}$ & $\begin{array}{c}\text { Chr06:17237912 } \\
\ldots 17245337(-)\end{array}$ & 7426 & 2433 & 810 & 92.26 & 5.99 & 42.23 & 89.10 & -0.249 \\
\hline PgSUS4 & $\begin{array}{l}\text { XM_031 } \\
516902.1\end{array}$ & $\begin{array}{c}\text { Chr08:5567456 } \\
\ldots .5574103(-)\end{array}$ & 6648 & 2418 & 805 & 92.54 & 6.09 & 32.35 & 92.87 & -0.278 \\
\hline PgSUS5 & $\begin{array}{l}\text { XM_031 } \\
551757.1\end{array}$ & $\begin{array}{c}\text { Chr08:24807424 } \\
\ldots .24812192(-)\end{array}$ & 4769 & 2706 & 901 & 102.58 & 8.19 & 39.61 & 83.77 & -0.348 \\
\hline
\end{tabular}

2 CDS, Coding DNA sequence; MW, molecular weight; pI, isoelectric point; Ai, aliphatic index;

3 GRAVY, grand average of hydropathicity. 
Table 2 (on next page)

Table 2. Secondary structural statistics of the PgSUS proteins 
1

2 Table 2. Secondary structural statistics of the PgSUS proteins

\begin{tabular}{ccccc}
\hline Protein & Alpha helix (\%) & Extended Beta strand (\%) & $\begin{array}{c}\text { Beta turn } \\
\mathbf{( \% )}\end{array}$ & Random coil (\%) \\
\hline PgSUS1 & 53.97 & 12.78 & 7.82 & 25.73 \\
PgSUS2 & 52.76 & 12.26 & 6.13 & 28.85 \\
PgSUS3 & 52.96 & 13.21 & 6.67 & 27.16 \\
PgSUS4 & 53.42 & 13.04 & 6.83 & 26.71 \\
PgSUS5 & 49.72 & 12.32 & 5.22 & 32.74 \\
\hline
\end{tabular}

3 


\section{Figure 1}

Figure 1 Phylogenetic relationship analysis of SUSs from pomegranate and seven other species

The phylogenetic relationship was analyzed by MEGA X program based on the ML method $J T+G+I$ and 1,000 bootstrap replications. The block lines and orange, green, and blue arcs indicate the members in subgroups SUS I, SUS II, and SUS III, respectively. PgSUS1 to PgSUS5 are highlighted in red dots. The species names are abbreviations as follows: At, Arabidopsis thaliana; Os, Oryza sativa; Gm, Glycine max; Md, Malus domestica; Pbr, Pyrus bretschneideri; $\mathrm{Pg}$, Punica granatum; Vv, Vitis vinifera, and Pp, Prunus persica. 


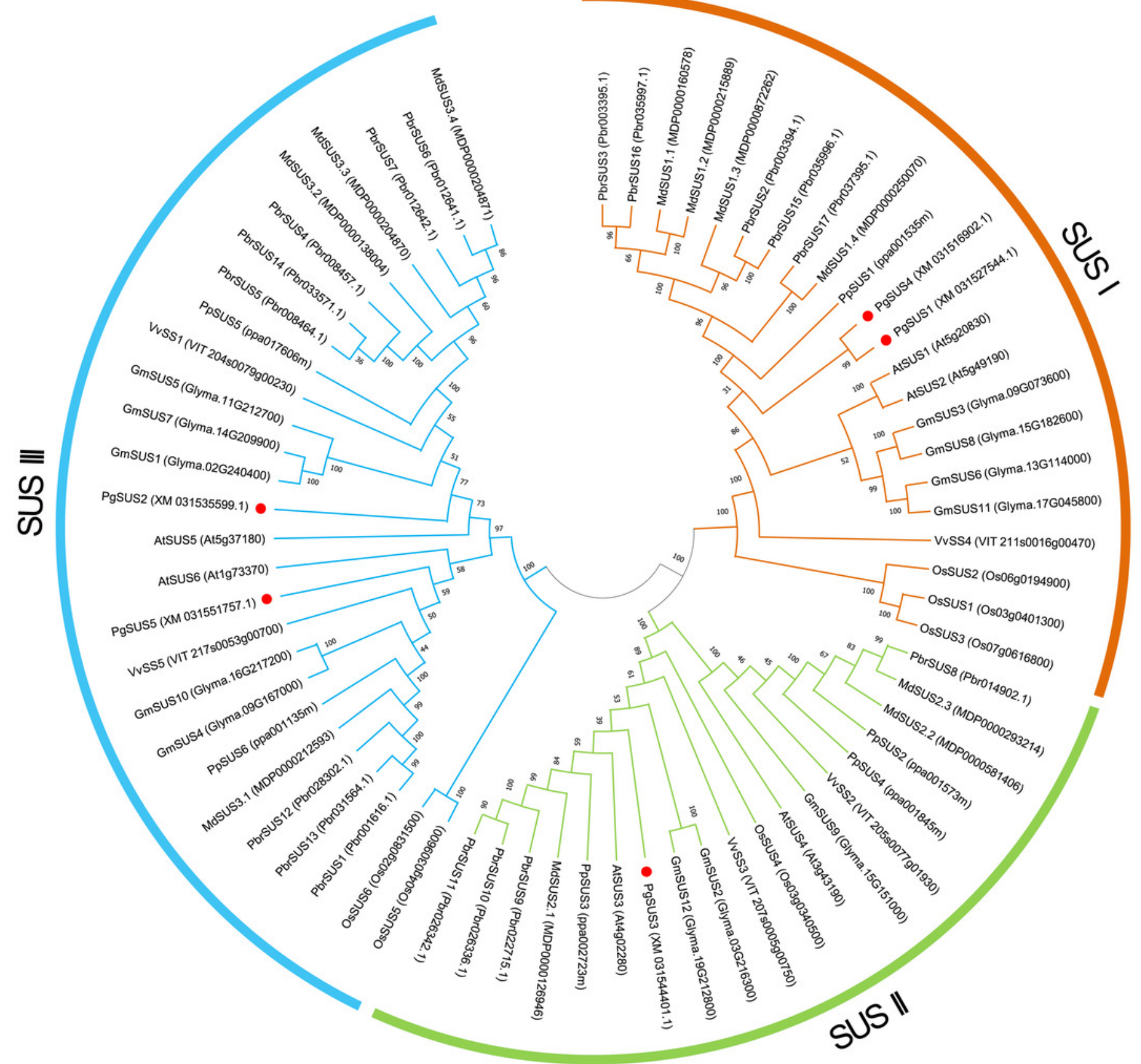




\section{Figure 2}

Figure 2 Analysis of gene structure, conserved motif, and domain of SUS genes family in seven species

(A) Exon/intron genomic structure of SUS genes. Exons, introns and untranslated regions (UTRs) are indicated by black rectangles, blue thin lines and green rectangles, respectively. (B) Composition and arrangement of the conserved motifs of SUS protein. Different colors and the numbers of the rectangles represent different motifs in the corresponding position of each SUS proteins. (C) Conserved domain structures of the SUS protein. The full-length protein sequences are indicated by thin black lines. The gene names PgSUS1 to PgSUS5 are highlighted in red. 


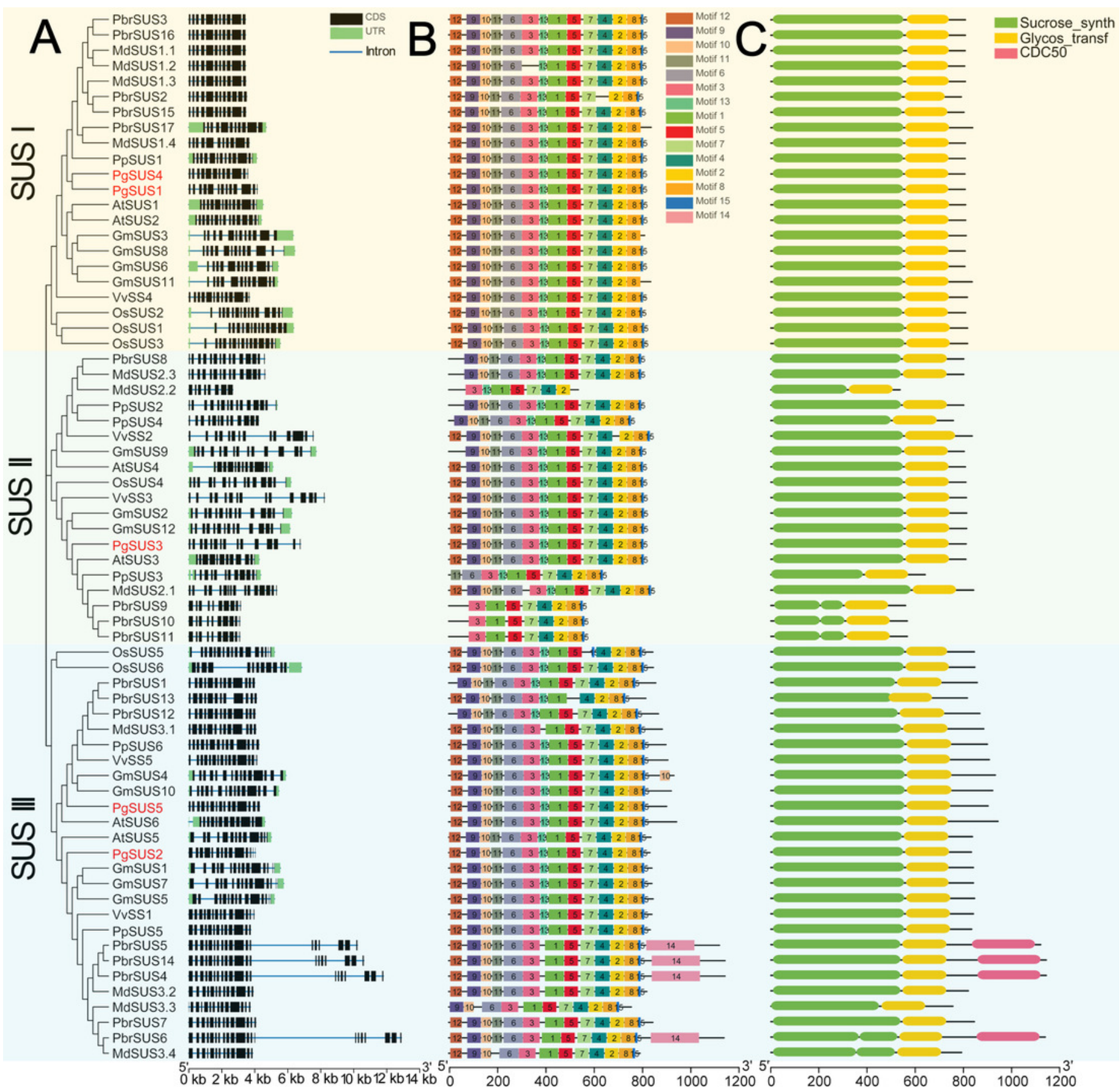




\section{Figure 3}

Figure 3 Synteny analysis of PgSUSs genes with other four species

The chromosomes of five species are marked with different colors. The short black lines on the circles represent the approximate positions of SUS genes of each species. Gene pairs with syntenic relationships are joined by red lines. The scale bar on the chromosome indicates chromosome length (Mb). 


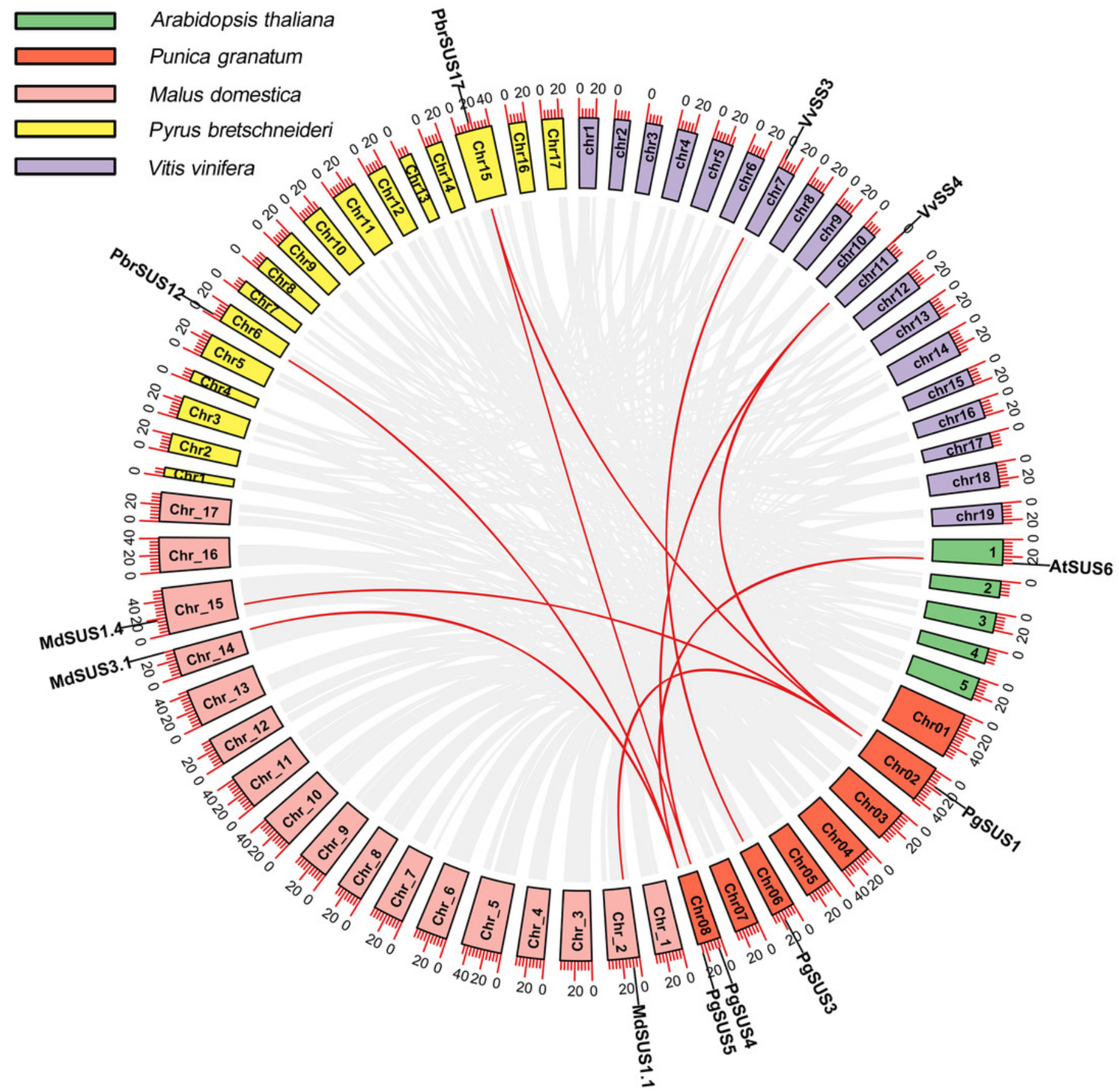




\section{Figure 4}

Figure 4 Analysis of cis-acting element numbers in promoter region of five PgSUS

Cis-acting elements of PgSUS genes were classified into five groups, including hormone responsive elements (HRE), tissue specific elements (TSE), light responsive elements (LRE), stress responsive elements (SRE), and other responsive elements (ORE). (A) Proportion of each functional group of cis-acting elements; (B) Number of cis-acting elements belonging to each functional group in individual PgSUS promoter sequences.
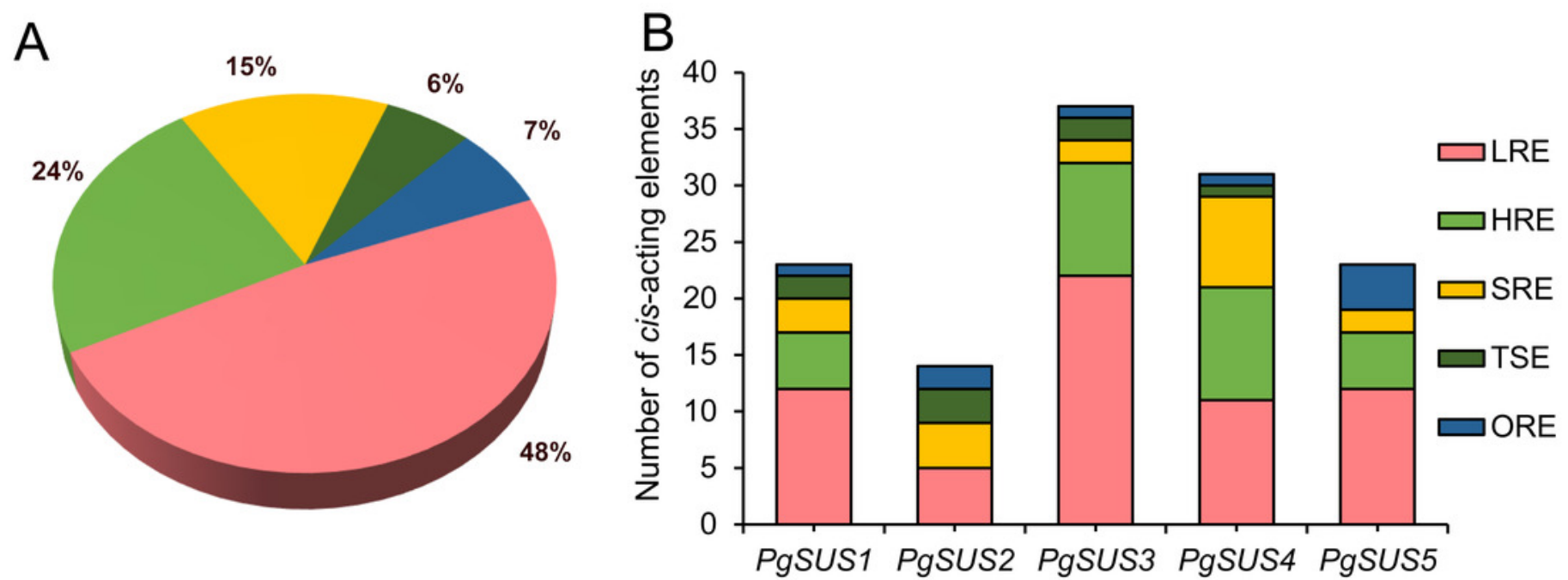


\section{Figure 5}

Figure 5 Expression analysis of the PgSUS genes in different tissues of pomegranate

(A) Expression profile of PgSUS genes in different organs or tissues of pomegranate, including root, leaf, flower, three stages of the pericarp, and the inner and outer seed coats $(50,95$, and 140 DAF). (B) Expression profile of PgSUS genes at different developmental stages of the seed coats in cultivated pomegranate cultivars 'Dabenzi' and 'Tunisia'. The number represents the number of days after flowering (DAF). Expression levels are depicted in different colors based on Log $_{2}$-transformed TPM +1 . White and red represent low and high expression levels, respectively.

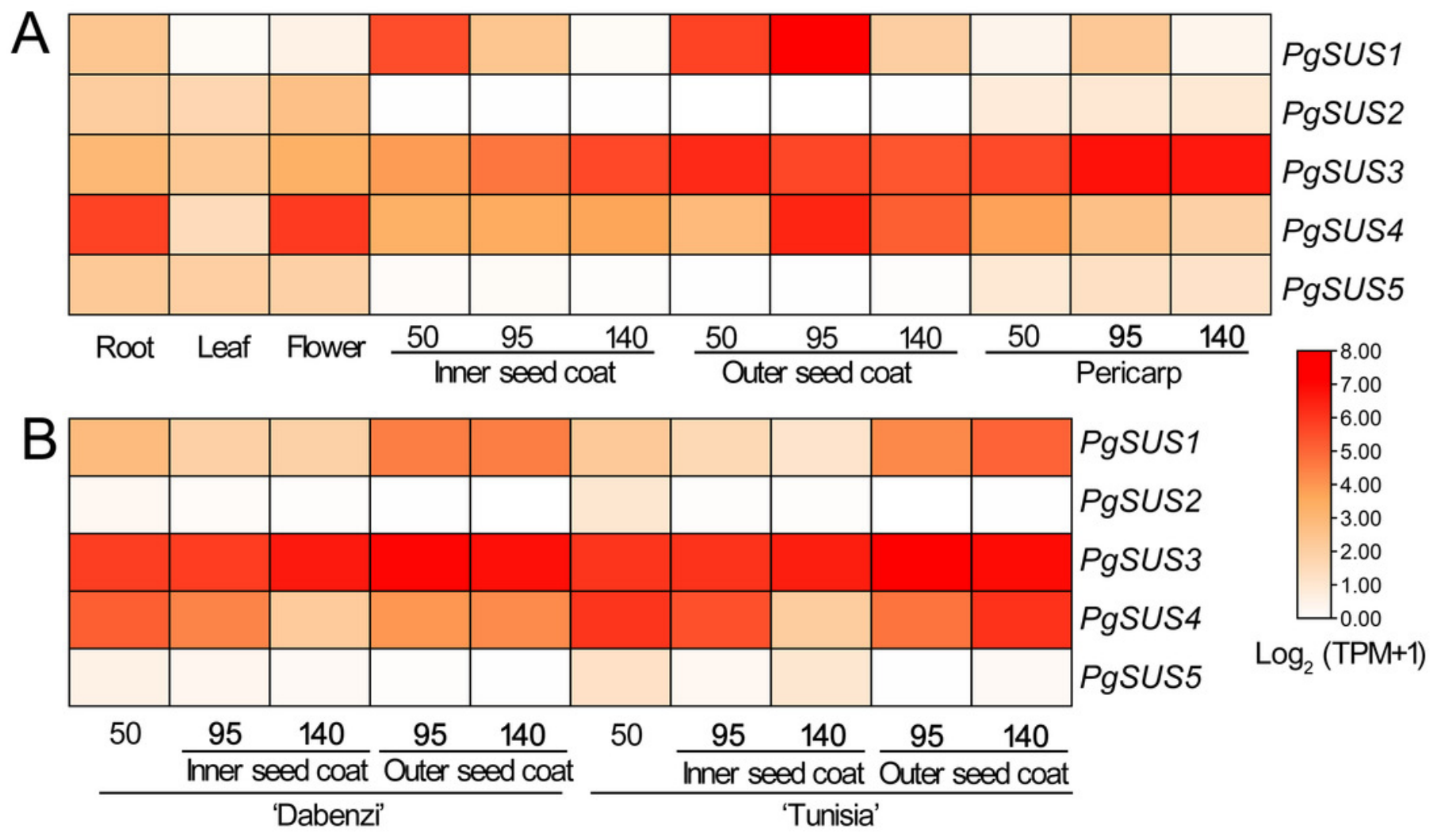


Figure 6

Figure 6 Expression pattern of five SUS genes assayed by qPCR

PgActin served as the reference gene. Gene expression was normalized to the leaf expression level, which was assigned with a value of 1 . Data represent the average of three independent replicates. Standard errors are shown as bars above columns. The different letters indicate significant differences at $p<0.05$.
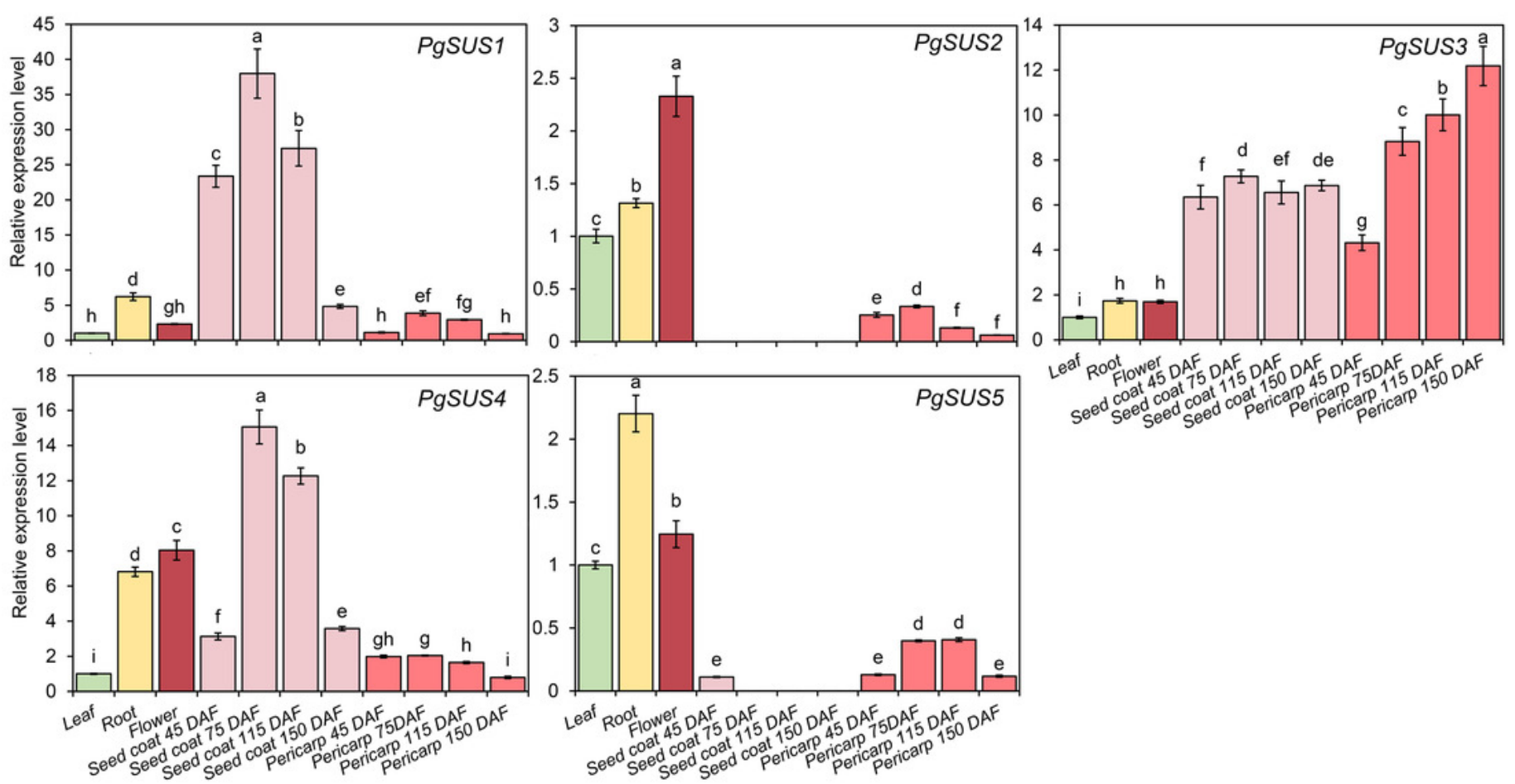